\title{
Comparison of search templates for gravitational waves from binary inspiral
}

\author{
Thibault Damour \\ Institut des Hautes Etudes Scientifiques, 91440 Bures-sur-Yvette, France \\ Bala R. Iyer \\ Raman Research Institute, Bangalore 560 080, India \\ B. S. Sathyaprakash \\ Department of Physics and Astronomy, Cardiff University, P.O. Box 913, Cardiff CF2 3YB, United Kingdom
}

(Received 3 October 2000; published 30 January 2001)

\begin{abstract}
We compare the performances of the templates defined by three different types of approaches: traditional post-Newtonian templates (Taylor approximants), "resummed" post-Newtonian templates assuming the adiabatic approximation and stopping before the plunge $(P$ approximants), and further "resummed" postNewtonian templates going beyond the adiabatic approximation and incorporating the plunge with its transition from the inspiral (effective-one-body approximants). The signal to noise ratio is significantly enhanced (mainly because of the inclusion of the plunge signal) by using these new effective-one-body templates relative to the usual post-Newtonian ones for a total binary mass $m \gtrsim 30 M_{\odot}$, and reaches a maximum around $m \sim 80 M_{\odot}$. Independently of the question of the plunge signal, the comparison of the various templates confirms the usefulness of using resummation methods. The paper also summarizes the key elements of the construction of various templates and thus can serve as a resource for those involved in writing inspiral search software.
\end{abstract}

DOI: 10.1103/PhysRevD.63.044023

PACS number(s): 04.30.Db, 04.25.Nx, 04.80.Nn, 95.55.Ym

\section{INTRODUCTION}

The late stage evolution in a compact binary, when the component stars are under the influence of the strong gravitational fields of each other and are moving at relativistic speeds, is dictated by the non-linear dynamics of general relativity and is very difficult to model. In the early stages of adiabatic inspiral (that is, when the inspiral time scale is much larger than the orbital time scale) it is possible to treat the problem of motion perturbatively and to expand the general relativistic equations of motion and wave generation formulas in a power series in $v / c, v$ being a characteristic velocity. (We henceforth use units such that $G=c=1$.) However, the phasing of the gravitational wave $(\mathrm{GW})$ signal derived from these perturbative results becomes increasingly inadequate as the two bodies approach each other. The characteristic velocity $v_{p}(m) \equiv\left(\pi m f_{p}\right)^{1 / 3}$, corresponding to the peak of the detector sensitivity to the inspiral signal from a binary of total mass $m=m_{1}+m_{2}$, is numerically equal [for the initial Laser Interferometric Gravitational Wave Observatory (LIGO), for which $\left.f_{p}=126 \mathrm{~Hz}\right]$ to $v_{p}(m)$ $=0.125\left(\mathrm{~m} / \mathrm{M}_{\odot}\right)^{1 / 3}$. For a double neutron star system one already has $v_{p}(2.8)=0.176$, while, for an archetypal $\left(10 M_{\odot}, 10 M_{\odot}\right)$ double black hole system one has $v_{p}(20)$ $=0.340$, quite close to the velocity corresponding to the last stable orbit $(\mathrm{LSO}): v_{\mathrm{LSO}} \approx 1 / \sqrt{6}=0.408$.

The present theoretical understanding has enabled the perturbative computation (via post-Newtonian expansions) of

\footnotetext{
${ }^{1}$ It should be noted that the LIGO noise curve used in this paper is the currently best available and different from that used in DamourIyer-Sathyaprakash 1 (DIS1) and DIS2.
}

the binary orbit and GW phase to an order $v^{5}$ beyond the standard quadrupole formula. We shall use these $v^{5}$-accurate results in this work. At present, we cannot count on the (hopeful) extension of the post-Newtonian (PN) perturbative calculations by another two orders, to order $v^{7}$, because currently used PN techniques leave undetermined a physically crucial parameter entering at the $v^{6}$ level [1-3]. Moreover, as emphasized some time ago [4], the PN series (which is essentially a Taylor expansion in powers of $v$ ) is a rather poorly convergent expansion. More precisely, if one considers the PN expansion of the crucial GW flux (see, e.g., Fig. 3 of [5]), one notices that the $v^{4}$-accurate and $v^{5}$-accurate approximations start significantly deviating, in opposite directions, from the exact (test-mass) result when $v \gtrsim 0.2$. As such relatively high values of $v$ are typically involved in the calculation of the GW phasing $\left[v \sim v_{p}(m) \geqslant 0.18\right.$ as soon as $\left.m \geqslant 2.8 M_{\odot}\right]$, one has to worry that search templates based on a straightforward use of PN-expanded results might be inadequate for the detection and/or measurement of inspiral signals, especially for the more massive systems $[\mathrm{m}$ $\geqslant 10 M_{\odot}$ implies $\left.v_{p}(m) \geqslant 0.34\right]$ which are likely to be the first potentially detectable events.

To address this crucial problem, we have been advocating [5-7] a new philosophy for making the optimal use of existing $\mathrm{PN}$ results, namely, to use several re-summation techniques to improve the convergence of the PN series, before using them to compute the GW phasing. As of now, we have proposed and studied three successive stages in the definition and use of such re-summation techniques. First, we constructed [5] time-domain signals, called $P$ approximantsstarting from the standard PN Taylor representation-which possess better convergence properties and capture the expected analytical behavior (poles and zeroes) of the relevant 
physical quantities quite well. We have shown that these new signal models, when compared with standard PN signals, are both more effectual (larger overlaps) and more faithful (smaller biases in the estimation of parameters) representations of some fiducial "exact" signals. Though time-domain $P$ approximants are better signal models than the standard "Taylor" approximants, they are computationally expensive to use in a data analysis exercise that searches for inspiral signals using hundreds of thousands of templates, which have to be correlated, with arbitrary time lags, with the detector output. This data-analysis computational cost is much reduced [thanks to the existence of efficient fast Fourier transform (FFT) algorithms] when one disposes of explicit analytic expressions for the Fourier transform of the templates.

Second, we found [6] explicit frequency-domain representations of $P$ approximants (as well as of standard PN templates) that are computationally inexpensive and are yet as faithful and effectual as the original time-domain models. This frequency-domain representation incorporates the "edge oscillations" due to the (assumed) abrupt shutoff of the time-domain signal occurring soon after the binary crosses the last stable orbit. In [6] we emphasized that the signal to noise (SNR) ratio of the first interferometric detectors is large enough for detection only for massive binary black hole systems of total mass $m \geq 25 M_{\odot}$. For such systems the characteristic velocity corresponding to the peak of the detector sensitivity is $v_{p}(25) \simeq 0.37$, which is very close to $v_{\mathrm{LSO}} \simeq 1 / \sqrt{6}$, the velocity at the last stable orbit. One, therefore, expects that the first detections are most likely to concern massive ${ }^{2}$ systems $\left(20 M_{\odot} \lesssim m \lesssim 40 M_{\odot}\right)$ with $v_{p}$ $\sim v_{\text {LSO }}$.

It is therefore crucial to push the re-summation techniques introduced in [5] further so as to be able to describe not only the GW phasing during the last cycles before LSO crossing, but also during the transition ${ }^{3}$ between inspiral and plunge, and during the plunge itself. Recently, Buonanno and Damour [7] combined some of the re-summation techniques of [5] and [6] with a novel approach to the general relativistic dynamics of two-body systems [8] to devise an improved type of re-summation approach to the GW phasing of coalescing binaries, able to describe in more detail the transition between inspiral and plunge. This "effective one-body" approach is the first one that goes beyond the "adiabatic approximation," used both in standard (non-resummed) PN approximants and in $P$ approximants.

The data analysis of inspiral, merger and ring-down was pioneered by Flanagan and Hughes [9]. They treated the problem of inspiral rather accurately but the merger and

\footnotetext{
${ }^{2}$ Note that, even for less massive systems, the necessity to capture more than $96.5 \%$ of the SNR, corresponding to a loss in the number of events by no more than $10 \%$, implies that one must accurately control the phasing of the waveform at frequencies significantly higher than $f_{p}$, corresponding to velocities significantly higher than $v_{p}(m)$.

${ }^{3}$ The $P$ approximants model this transition by a sharp cutoff in the signal.
}

plunge were treated by assuming that about $10 \%$ of the rest mass energy would be emitted during merger. This quite optimistic estimate was based on a crude model of the coalescence of maximally spinning black holes, and was arbitrarily extended to all cases. A similar back-of-the-envelope consideration of the ring-down amplitude let them to optimistically assume that about $3 \%$ of the rest mass energy would be emitted during ring-down.

In this paper we discuss only non-spinning ${ }^{4}$ binaries and we make no ad hoc assumption about the total energy radiated during the merger phase. The effective one-body (EOB) formalism does not treat the inspiral and plunge phases separately. Indeed, in this formalism the plunge is seen as a natural continuation of the inspiral phase contributing (for equal masses) about 0.6 orbital cycles (or $1.2 \mathrm{GW}$ cycles), with a total energy associated with the plunge around $0.7 \%$. The energy emitted during the following (matched) ring-down phase is also found to be around $0.7 \%$ [11]. These energy losses are much smaller than the Flanagan-Hughes "guesstimates" of $10 \%$ and $3 \%$, respectively. Consequently, it is unlikely that we will be able to detect the plunge phase of the EOB waveforms separately, irrespective of the mass of the system. This is in sharp contrast to the Flanagan-Hughes claim that the SNR contribution of the sole merger phase of massive black holes of total mass in the range $30-1000 M_{\odot}$ will dominate over the inspiral phase contribution. Note also that (for a source at $100 \mathrm{Mpc}$ ) the (merger-dominated) SNR of [9] reaches a maximum of 40 around $m \sim 200 M_{\odot}$, while our (inspiral-dominated) SNR reaches a maximum of 8 around $m \sim 80 M_{\odot}$. [It seems that most of the difference between Fig. 4 of Flanagan and Hughes and our Fig. 1 below, e.g. a factor of 3 (between their 25 and our 8) between the SNR for a $80 M_{\odot}$ source at $100 \mathrm{Mpc}$, comes from the huge difference in energy loss during merger.]

In this paper we make a prediction that the merger phase will enhance the inspiral phase SNR by about $10 \%$ for $m$ $\sim 30 M_{\odot}$ and by about $300 \%$ for $m \sim 80 M_{\odot}$. Our best candidate sources are stellar mass black hole binaries of total mass in the range $30-90 M_{\odot}$. The inspiral phase is the dominant signal for most of this range, merger being important for only the heavier systems. We also conclude that the ringdown phase is in itself not a significant contribution for $m$ $\lesssim 200 M_{\odot}$.

There is one word of caution regarding the plunge signal: Even though the plunge lasts for only about half an orbital cycle, its spectral content spreads over a large frequency range. Consequently, the number of frequency bins over which the signal spreads out is quite large and it is not advisable to use a non-optimal method to try to detect the plunge part in isolation. In fact, we believe that one of the robust predictions of the EOB approach (at least in the case of slowly spinning holes) is that the plunge signal is a smooth continuation of the inspiral one and that one should use templates that are phase coherent all over the inspiral-

\footnotetext{
${ }^{4}$ In view of current black hole binary formation mechanisms [10], we think it likely that most of them will include only slowly spinning holes.
} 
plus-plunge phase. We are aware of the approximate nature of the EOB results (especially beyond the LSO) and do not claim that the EOB waveform is the last word on the problem, but our position is the following: (i) in absence of comparatively accurate alternative results, it is important to study in detail the predictions coming from the EOB waveform, and (ii) we shall finally recommend to use a bank of filters which cover a large range of possibilities, with special weight being given to the best-tested "resummed" templates.

We also hope that our work will give an additional incentive to numerical relativity groups toward computing waveforms which are at least as accurate (and physically complete) as the EOB one. In particular, let us recall that [7] has proposed a new approach to the numerical computation of binary black hole coalescences: namely, to start the numerical evolution just after LSO crossing, i.e. at a stage where one can still trust the resummed PN estimate of the dynamics of two black holes, but where there is only 0.6 orbit to evolve before coalescence. To this aim Ref. [7] has provided explicit results for the initial dynamical data [positions and momenta in Arnowitt-Deser-Misner (ADM) coordinates] of this problem. However, apart from stimulating further thoughts on the problem [12], we are not aware of the existence of numerical simulations implementing the proposal of Ref. [7], nor are we aware of other numerical work leading to explicit (non-adiabatic) waveforms for coalescing binaries which could be compared to the EOB one.

Data analysis groups associated with various groundbased interferometers are now finalizing the analysis software that will be used for GW searches in data that are expected to become available in a few years time. It is essential that these groups be aware of recent theoretical developments and of their respective merits so as to take the best advantage of the current knowledge in writing their software. With this view in mind the aim of this paper is twofold: First, we wish to compare the performances of the templates defined by the three different types of approaches mentioned above (traditional "non-resummed" PN templates, "resummed" PN templates assuming the adiabatic approximation and stopping before the plunge, and further "resummed" PN templates going beyond the adiabatic approximation and incorporating the plunge with its transition from the inspiral.) Second, in view of the fact that the original publications $[5,6]$ are quite complex and technical, we wish to summarize in a more accessible manner the key elements of the techniques introduced there (and re-used, with further inputs, in [7].) The present work should serve as an easily accessible resource for data analysis groups. Readers interested in a more detailed understanding of our general approach are referred to [5-8] for motivation, formalism, logical reasoning, exhaustive tests and further discussion of the new signal models.

\section{TIME-DOMAIN PHASING FORMULAS IN THE ADIABATIC APPROXIMATION}

In searching for GWs from an inspiraling compact binary we are faced with the following data analysis problem: On the one hand, we have some (unknown) exact gravitational waveform $h^{X}\left(t ; \lambda_{k}\right)$ where $\lambda_{k}, k=1, \ldots, n_{\lambda}$, are the parameters of the signal (e.g., the masses $m_{1}$ and $m_{2}$ of the members of the emitting binary). On the other hand, we have theoretical calculations of the motion of [13,1-3] and gravitational radiation from $[14,15]$ binary systems consisting of neutron stars (NSs) or black holes (BHs) giving the PN expansions of an energy function $E\left(x \equiv v^{2}\right)$, which is related to the total relativistic energy $E_{\text {tot }}$ via $E_{\text {tot }}=\left(m_{1}+m_{2}\right)(1+E)$, and a GW luminosity (or "flux") function $\mathcal{F}(v)$. Here, the dimensionless argument $v \equiv x^{1 / 2}$ is an invariantly defined "velocity" related to the instantaneous GW frequency $F$ (=twice the orbital frequency) by $v \equiv(\pi m F)^{1 / 3}$. Given PN expansions of the motion of and gravitational radiation from a binary system, one needs to compute the "phasing formula," i.e. an accurate mathematical model for the evolution of the GW phase $\phi^{\mathrm{GW}}=p\left[t ; \lambda_{i}\right]$, involving the set of parameters $\left\{\lambda_{i}\right\}$ carrying information about the emitting binary system. In the adiabatic approximation the phasing formula is easily derived from the energy and flux functions. Indeed, the standard energy-balance equation $d E_{\text {tot }} / d t=-\mathcal{F}$ gives the following parametric representation of the phasing formula:

$$
\begin{aligned}
& t(v)=t_{\text {ref }}+m \int_{v}^{v_{\text {ref }}} d v \frac{E^{\prime}(v)}{\mathcal{F}(v)}, \\
& \phi(v)=\phi_{\text {ref }}+2 \int_{v}^{v_{\text {ref }}} d v v^{3} \frac{E^{\prime}(v)}{\mathcal{F}(v)},
\end{aligned}
$$

where $t_{\text {ref }}$ and $\phi_{\text {ref }}$ are integration constants and $v_{\text {ref }}$ an arbitrary reference velocity. [It is sometimes convenient, though by no means necessary, to take as $v_{\text {ref }}$ the velocity $v$ at the last stable orbit (see below).] From the viewpoint of computational purposes it is more efficient to work with the following pair of coupled, non-linear, ordinary differential equations (ODEs), which are equivalent to the above parametric formulas:

$$
\frac{d \phi}{d t}-\frac{2 v^{3}}{m}=0, \quad \frac{d v}{d t}+\frac{\mathcal{F}(v)}{m E^{\prime}(v)}=0 .
$$

We shall see later that, for massive systems, the adiabatic approximation fails and one must replace the two ODEs by a more complicated ODE system. We now turn to a discussion of what is known about the two functions $E(v)$ and $\mathcal{F}(v)$ entering the phasing formula and how that knowledge can be improved.

\section{A. $T$ approximants}

We denote by $E_{T_{n}}$ and $\mathcal{F}_{T_{n}}$ the $n^{\text {th }}$-order ${ }^{6}$ "Taylor" approximants (as defined by the PN expansion) of the energy and flux functions:

\footnotetext{
${ }^{5}$ We work within the "restricted" waveform approximation which keeps only the leading harmonic in the GW signal.

${ }^{6}$ The label $n$ always refers to an approximant accurate up to $v^{n}$ $=x^{(n / 2)}$ included.
} 
TABLE I. Taylor coefficients of the energy functions $E_{T_{n}}(x)$ and $e_{T_{n}}(x)$ and the corresponding location of the LSO and pole. As there are no terms of order $v^{2 k+1}$ we have exceptionally chosen (for this table only) the expansion parameter to be $x \equiv v^{2}$ rather than $v$. In all cases the $k=0$ coefficient is equal to 1 , the last stable orbit is defined only for $k \geqslant 1$ in the case of $T$ approximants and for $k \geqslant 2$ in the case of $P$ approximants and $N$ denotes the "Newtonian value."

\begin{tabular}{cccc}
\hline \hline$k$ & $N$ & 1 & 2 \\
\hline$E_{k}$ & $-\frac{\eta v^{2}}{2}$ & $-\frac{9+\eta}{12}$ & $-\frac{81-57 \eta+\eta^{2}}{24}$ \\
$e_{k}$ & $-x\left(=-v^{2}\right)$ & $-\frac{3+\eta}{3}$ & $-\frac{36-35 \eta}{12}$ \\
$e_{P_{k}}$ & $-x\left(=-v^{2}\right)$ & $c_{1}=\frac{3+\eta}{3}$ & $c_{2}=-\frac{144-81 \eta+4 \eta^{2}}{36+12 \eta}$ \\
$x_{T_{k}}^{\text {LSO }}$ & - & $\frac{6}{9+\eta}$ & $-E_{1}+\left(E_{1}^{2}-3 E_{2}\right)^{1 / 2}$ \\
$x_{P_{k}}^{\text {LSO }}$ & - & - & $\frac{-1+\left(-c_{1} / c_{2}\right)^{1 / 2}}{c_{1}+c_{2}}$ \\
$x_{P_{k}}^{\text {pole }}$ & - & - & $\frac{4(3+\eta)}{36-35 \eta}$ \\
\hline \hline
\end{tabular}

$$
\begin{aligned}
& E_{T_{2 n}}(x) \equiv E_{N}(x) \sum_{k=0}^{n} \hat{E}_{k}(\eta) x^{k} \\
& \mathcal{F}_{T_{n}}(x) \equiv \mathcal{F}_{N}(x)\left[\sum_{k=0}^{n} \hat{\mathcal{F}}_{k}(\eta) v^{k}+\sum_{k=6}^{n} \hat{L}_{k}(\eta) \log \left(v / v_{0}\right) v^{k}\right]
\end{aligned}
$$

where

$$
E_{N}(x)=-\frac{1}{2} \eta x, \quad \mathcal{F}_{N}(x)=\frac{32}{5} \eta^{2} x^{5}
$$

Here the subscript $N$ denotes the "Newtonian value," $\eta$ $\equiv m_{1} m_{2} / m^{2}$ is the symmetric mass ratio, and $v_{0}$ is a fiducial constant to be chosen below. In the test mass limit, i.e. $\eta$ $\rightarrow 0, E(x)$ is known exactly, from which the Taylor expansion of $E_{T_{n}}(v, 0)$ in Eq. (2.3), can be computed to all orders. In the $\eta \rightarrow 0$ limit, the exact flux is known numerically $[16,17]$ and the Taylor expansion of flux in Eq. (2.3) is known [17-19] up to order $n=11$. On the other hand, in the physically relevant case where $\eta$ is finite, the above Taylor approximants are known $[14,15]$ only up to five-halves PN order, i.e. $n=5$. Recently the energy function has been computed up to $3 \mathrm{PN}$ order, i.e., $n=6$, though with the presence of an unknown parameter [2,3]. All the completely known coefficients in the expansions are enlisted in Table I.

The problem is to construct a sequence of approximate waveforms $h_{n}^{A}\left(t ; \lambda_{k}\right)$, starting from the PN expansions of $E(v)$ and $\mathcal{F}(v)$. In formal terms, any such construction defines a map from the set of the Taylor coefficients of $E$ and $\mathcal{F}$ into the (functional) space of waveforms. Up to now, the literature has considered (one of) the $\operatorname{map}(\mathrm{s})$, say $T$,

$$
\left(E_{T_{n}}, \mathcal{F}_{T_{n}}\right) \stackrel{T}{\rightarrow} h_{n}^{T}\left(t, \lambda_{k}\right),
$$

obtained by inserting the successive Taylor approximants into the phasing formula $[4,16]$. For brevity, we often refer to these "Taylor"' approximants as " $T$ approximants." It must also be emphasized that even within this Taylor family of templates, there are at least three ways of proceeding further, leading to the following three inequivalent constructs:

(t1) One can retain the rational polynomial $\mathcal{F}_{T_{n}} / E_{T_{n}}$ as it appears in Eq. (2.2) and integrate the two ODEs numerically. We shall denote the phasing formula so obtained as $\phi_{T_{n}}^{(1)}(t)$ :

$$
\frac{d \phi^{(1)}}{d t}-\frac{2 v^{3}}{m}=0, \quad \frac{d v}{d t}+\frac{\mathcal{F}_{T_{n}}(v)}{m E_{T_{n}}^{\prime}(v)}=0 .
$$

(t2) One can re-expand the rational function $\mathcal{F}_{T_{n}} / E_{T_{n}}$ appearing in the phasing formula and truncate it at order $v^{n}$, in which case the integrals in Eq. (2.1) can be worked out analytically, to obtain a parametric representation of the phasing formula in terms of polynomial expressions in the auxiliary variable $v$ :

$$
\begin{aligned}
& \phi_{T_{n}}^{(2)}(v)=\phi_{\mathrm{ref}}^{(2)}+\phi_{N}^{v}(v) \sum_{k=0}^{n} \hat{\phi}_{k}^{v} v^{k}, \\
& t_{T_{n}}^{(2)}(v)=t_{\mathrm{ref}}^{(2)}+t_{N}^{v}(v) \sum_{k=0}^{n} \hat{t}_{k}^{v} v^{k},
\end{aligned}
$$

where a superscript on the coefficients (e.g. $\phi_{1}^{v}$ ) indicates that $v$ is the expansion parameter (as is explicit from Table II, the coefficient of $\phi_{k}^{v}$ include in some cases, a $\log v$ dependence).

(t3)Finally, the second of the polynomials in Eq. (2.7) can be inverted and the resulting polynomial for $v$ in terms of $t$ can be substituted in $\phi^{(2)}(v)$ to arrive at an explicit timedomain phasing formula

$\phi_{T_{n}}^{(3)}(t)=\phi_{\text {ref }}^{(3)}+\phi_{N}^{t} \sum_{k=0}^{n} \hat{\phi}_{k}^{t} \theta^{k}, \quad F_{T_{n}}^{(3)}(t)=F_{N}^{t} \sum_{k=0}^{n} \hat{F}_{k}^{t} \theta^{k}$,

where $\quad \theta=\left[\eta\left(t_{\text {ref }}-t\right) /(5 m)\right]^{-1 / 8} \quad$ and $\quad F \equiv d \phi / 2 \pi d t$ $=v^{3} /(\pi m)$ is the instantaneous GW frequency. The coefficients in these expansions are all listed in Table II.

\section{B. $P$ approximants}

Before defining new "resummed" energy and flux functions with improved performances we digress for a brief reminder of Padé re-summation, which is a standard mathematical technique used to accelerate the convergence of poorly converging power series. Let $S_{n}(v)=a_{0}+a_{1} v+\cdots$ $+a_{n} v^{n}$ be a truncated Taylor series. A Padé approximant of the function whose Taylor approximant to order $v^{n}$ is $S_{n}$ is 
TABLE II. Taylor coefficients of the flux, phase, time and frequency. $N$ denotes the "Newtonian value" and $\theta=\left[\eta\left(t_{\mathrm{LSO}}\right.\right.$ $-t) /(5 m)]^{-1 / 8}$. In all cases the $k=0$ coefficient is 1 and the $k=1$ coefficient is zero. In certain cases the 2.5 PN term involves a $v^{5} \log v$ or $\theta^{5} \log \theta$ term rather than a $v^{5}$ or $\theta^{5}$ term. In those cases we conventionally include the $\log v$ dependence in the listed coefficient. Chirp parameters $\tau_{k}, k \geqslant 1$, are related to the expansion parameters $t_{k}^{v}$ and $\phi_{k}^{v}$ via $\tau_{k}=\left(8 \phi_{k}^{v}-5 t_{k}^{v}\right) / 3$. We have given the simplified expressions for these in all cases, except $k=5$ where no simplification occurs due to the presence of the logarithm term in $\phi_{5}^{v}$.

\begin{tabular}{lccccc}
\hline \hline$k$ & $N$ & 2 & 3 & 5 \\
\hline $\mathcal{F}_{k}$ & $\frac{32 \eta^{2} v^{10}}{5}$ & $-\frac{1247}{336}-\frac{35 \eta}{12}$ & $4 \pi$ & $-\frac{44711}{9072}+\frac{9271 \eta}{504}+\frac{65 \eta^{2}}{18}$ & $-\left(\frac{8191}{672}+\frac{535 \eta}{24}\right) \pi$ \\
$t_{k}^{v}$ & $-\frac{5 m}{256 \eta v^{8}}$ & $\frac{743}{252}+\frac{11 \eta}{3}$ & $-\frac{32 \pi}{5}$ & $\frac{3058673}{508032}+\frac{5429 \eta}{504}+\frac{617 \eta^{2}}{72}$ & $-\left(\frac{7729}{252}+\eta\right) \pi$ \\
$\phi_{k}^{v}$ & $-\frac{1}{16 \eta v^{5}}$ & $\frac{3715}{1008}+\frac{55 \eta}{12}$ & $-10 \pi$ & $\frac{15293365}{1016064}+\frac{27145 \eta}{1008}+\frac{3085 \eta^{2}}{144}$ & $\left(\frac{38645}{672}+\frac{15 \eta}{8}\right) \pi \ln \left(\frac{v}{v_{\mathrm{lso}}}\right)$ \\
$\phi_{k}^{t}$ & $-\frac{2}{\eta \theta^{5}}$ & $\frac{3715}{8064}+\frac{55 \eta}{96}$ & $-\frac{3 \pi}{4}$ & $\frac{9275495}{14450688}+\frac{284875 \eta}{258048}+\frac{1855 \eta^{2}}{2048}$ & $\left(\frac{38645}{21504}+\frac{15 \eta}{256}\right) \pi \ln \left(\frac{\theta}{\theta_{\mathrm{lso}}}\right)$ \\
$F_{k}^{t}$ & $\frac{\theta^{3}}{8 \pi m}$ & $\frac{743}{2688}+\frac{11 \eta}{32}$ & $-\frac{3 \pi}{10}$ & $\frac{1855099}{14450688}+\frac{56975 \eta}{258048}+\frac{371 \eta^{2}}{2048}$ & $-\left(\frac{7729}{21504}+\eta\right) \pi$ \\
$\tau_{k}$ & $\frac{3}{128 \eta}$ & $\left.\frac{5}{9}\left(\frac{743}{84}\right)+11 \eta\right)$ & $-16 \pi$ & $2 \phi_{4}^{v}$ & $\frac{1}{3}\left(8 \phi_{5}^{v}-5 t_{5}^{v}\right)$ \\
\hline \hline
\end{tabular}

defined by two integers $m, k$ such that $m+k=n$. If $T_{n}[\cdots]$ denotes the operation of expanding a function in Taylor series and truncating it to accuracy $v^{n}$ (included), the $P_{k}^{m}$ Padé approximant of $S_{n}$ is defined by

$$
P_{k}^{m}(v)=\frac{N_{m}(v)}{D_{k}(v)} ; \quad T_{n}\left[P_{m}^{k}(v)\right] \equiv S_{n}(v),
$$

where $N_{m}$ and $D_{k}$ are polynomials in $v$ of order $m$ and $k$ respectively. If one assumes that $D_{k}(v)$ is normalized so that $D_{k}(0)=1$, i.e. $D_{k}(v)=1+q_{1} v+\cdots$, one shows that Padé approximants are uniquely defined by Eq. (2.9). In many cases the most useful ${ }^{7}$ Padé approximants are the ones near the "diagonal," $m=k$, i.e. $P_{m}^{m}$ if $n=2 m$ is even and $P_{m}^{m+1}$ or $P_{m+1}^{m}$ if $n=2 m+1$ is odd. In this work we shall use the diagonal $\left(P_{m}^{m}\right)$ and the "sub-diagonal" $\left(P_{m+1}^{m}\right)$ approximants which can be conveniently ${ }^{8}$ written in a continued fraction form [20]. For example, given $S_{2}(v)=a_{0}+a_{1} v$ $+a_{2} v^{2}$ one looks for

$$
P_{1}^{1}(v)=\frac{c_{0}}{1+\frac{c_{1} v}{1+c_{2} v}}=\frac{c_{0}\left(1+c_{2} v\right)}{1+\left(c_{1}+c_{2}\right) v}
$$

The continued fraction Padé coefficients of a series containing six terms, i.e. $S_{5}(v)$, are given by

\footnotetext{
${ }^{7}$ The rare theorems dealing with the Padé technique concern the convergence of "near-diagonal" Padé approximants, i.e. $m \rightarrow \infty$ with $|m-k|$ fixed.

${ }^{8} \mathrm{~A}$ convenience of this form is that the $n$th continued-fraction coefficient $c_{n}$ (see below) depends only on the knowledge of the PN coefficients of order $\leqslant n$.
}

$$
\begin{aligned}
c_{0}= & a_{0}, \quad c_{1}=-\frac{a_{1}}{a_{0}}, \quad c_{2}=-\frac{a_{2}}{a_{1}}+\frac{a_{1}}{a_{0}}, \\
c_{3}= & \frac{a_{0}\left(a_{1} a_{3}-a_{2}^{2}\right)}{a_{1}\left(a_{1}^{2}-a_{2} a_{0}\right)}, \\
c_{4}= & -\frac{c_{0} c_{1}\left(c_{2}+c_{1}\right)^{3}+c_{0} c_{1} c_{2} c_{3}\left(c_{3}+2 c_{2}+2 c_{1}\right)-a_{4}}{c_{0} c_{1} c_{2} c_{3}}, \\
c_{5}= & -\frac{\left[\left(c_{2}+c_{1}\right)^{2}+c_{2} c_{3}\right]^{2}}{c_{2} c_{3} c_{4}} \frac{\left(c_{4}+c_{3}+c_{2}+c_{1}\right)^{2}}{c_{4}} \\
& -\frac{a_{5}}{c_{0} c_{1} c_{2} c_{3} c_{4}} .
\end{aligned}
$$

In [5] and [6] we introduced several techniques for "resumming" the Taylor expansions (in powers of $v$ ) of the energy and flux functions. Starting from the PN expansions of $E$ and $\mathcal{F}$, in DIS1 we proposed a new class of waveforms, called $P$ approximants, based on two essential ingredients: (i) the introduction, on theoretical grounds, of two new, supposedly more basic and hopefully better behaved, energytype and flux-type functions, say $e(v)$ and $f(v)$, and (ii) the systematic use of Padé approximants (instead of straightforward Taylor expansions) when constructing successive approximants of the intermediate functions $e(v), f(v)$. Schematically, our procedure is based on the following map, say " $P$ ": 


$$
\begin{aligned}
\left(E_{T_{n}}, \mathcal{F}_{T_{n}}\right) & \rightarrow\left(e_{T_{n}}, f_{T_{n}}\right) \rightarrow\left(e_{P_{n}}, f_{P_{n}}\right) \\
& \rightarrow\left(E\left[e_{P_{n}}\right], \mathcal{F}\left[e_{P_{n}}, f_{P_{n}}\right]\right) \rightarrow h_{n}^{P}\left(t, \lambda_{k}\right) .
\end{aligned}
$$

Our new energy function $e(x)$, where $x \equiv v^{2}$, is constructed out of the total relativistic energy $E_{\text {tot }}(x)$ using

$$
e(x) \equiv\left(\frac{E_{\mathrm{tot}}^{2}-m_{1}^{2}-m_{2}^{2}}{2 m_{1} m_{2}}\right)^{2}-1 .
$$

The function $E(x)$ entering the phasing formulas is the total energy per unit mass after subtracting out the rest mass energy, $E(x)=\left[E_{\text {tot }}(x)-m\right] / m$, and is given in terms of $e(x)$ by

$$
\begin{gathered}
E(x)=\{1+2 \eta[\sqrt{1+e(x)}-1]\}^{1 / 2}-1, \\
\frac{d E}{d x}=\frac{\eta e^{\prime}(x)}{2[1+E(x)] \sqrt{1+e(x)}} .
\end{gathered}
$$

Note that the quantity $E^{\prime}(v)$, needed in the phasing formula, is related to $d E(x) / d x$ via $E^{\prime}(v)=2 v d E(x) / d x$. In the testmass limit $e(x)$ and $d E(x) / d x$ are known exactly:

$$
\begin{aligned}
e_{\eta=0}(x) & =-x \frac{1-4 x}{1-3 x}, \\
E^{\eta=0}(x) & =\eta\left(\frac{1-2 x}{\sqrt{1-3 x}}-1\right), \\
\frac{d E^{\eta=0}}{d x} & =-\frac{\eta}{2} \frac{(1-6 x)}{(1-3 x)^{3 / 2}} .
\end{aligned}
$$

The rationale for using $e(x)$ as the basic quantity rather than $E_{\text {tot }}(x)$ can be found in [5]; here we note the following two points: (1) In the test mass case $e(x)$ is meromorphic in the complex $x$ plane, with a simple pole singularity, while the function $E(x)$ is non-meromorphic and exhibits a branch cut. (2) Second, in the test mass case, the Padé approximant of $e_{T_{2 n}}(x)$, for $n \geqslant 2$, yields the known exact expression including the location of the LSO and the pole. Therefore, the function $e(x)$ is more suitable in analyzing the analytic structure than is $E(x)$. In the comparable mass case, under the assumption of structural stability between the case $\eta$ $\rightarrow 0$ and the case of finite $\eta$, one can expect the exact function $e(x)$ to admit a simple pole singularity on the real axis, $\propto\left(x-x_{\text {pole }}\right)^{-1}$. We do not know the location of this singularity, but Padé approximants are excellent tools for giving accurate representations of functions having such pole singularities [20].

Our proposal is the following: Given some usual Taylor approximant to the normal energy function, $E_{T_{2 n}}=$ $-\frac{1}{2} \eta x\left(1+E_{1} x+E_{2} x^{2}+\cdots+E_{n} x^{n}\right)$, one first computes the corresponding Taylor approximant for the $e$ function, say

$$
e_{T_{2 n}}(x)=-x \sum_{k=0}^{n} e_{k} x^{k}
$$

(Remember that we consistently label the successive approximants by the order in velocity; e.g. a $2 P N$-accurate object has the label 4.) Then, one defines the Padé approximant of $e_{T_{2 n}}(x),{ }^{9}$

$$
e_{P_{2 n}}(x) \equiv-x P_{m+\epsilon}^{m}\left[\sum_{k=0}^{n} e_{k} x^{k}\right]
$$

where $\epsilon=0$ or 1 depending on whether $n \equiv 2 m+\epsilon$ is even or odd. We shall call the continued fraction Padé coefficients of $e_{P_{2 n}}$ as $c_{1}, c_{2}, \ldots$ (note that $c_{0} \equiv 1$ ). They are given in terms of $e_{k}$ in Table I. Given a continued fraction approximant $e_{P_{2 n}}(x)$ of the truncated Taylor series $e_{T_{2 n}}$ of the energy function $e(x)$ the corresponding $E_{P_{2 n}}(x)$ and $d E_{P_{2 n}}(x) / d x$ functions are obtained using formulas in Eq. (2.14) by replacing $e(x)$ on the right hand side with $e_{P_{2 n}}(x)$.

Apart from using it to improve the convergence of the PN series, Ref. [5] has also proposed to use the Padé-resummed function $e_{P_{2 n}}(x)$ to determine the location of the LSO, the Padé estimates of the LSO being defined by considering the minima of $e_{P_{2 n}}(x)$. In contrast, in the Taylor case one must, for consistency, use the minima of $E_{T_{n}}(v)$ to define the locations of the LSO. We have confirmed that in the test mass case this Padé-based method yields the exact result at orders $v^{4}$ and beyond while the corresponding Taylor-based method [considering the minima of $E_{T_{n}}(v)$ ] gives unacceptably high estimates of $v_{\text {LSO }}$, i.e. of the GW frequency at the LSO. In the finite $\eta$ case, the Padé-resummed predictions are in good qualitative (and reasonable quantitative) agreement with the more recent predictions based on the "effective-one-body", approach [8]. The location of the LSOs for the various approximations and the location of the light ring [i.e. the pole singularity in $e_{P_{2 n}}(x)$ ] are also tabulated in Table I.

Having defined a new energy function, we move on to introduce a new flux function. The aim is to define an analytic continuation of the flux function to control its analytic structure as also to handle the logarithmic terms that appear in the flux function in Eq. (2.3). Factoring out the logarithmic terms is what allows us to use standard Padé techniques effectively in this problem.

It has been pointed out [19] that the flux function in the test mass case $\mathcal{F}(v ; \eta=0)$ has a simple pole at the light ring $v^{2}=1 / 3$. It has been argued that the origin of this pole is quite general [cf. [5], discussion following Eq. (4.3)] and that even in the case of comparable masses we should expect to have a pole singularity in $\mathcal{F}$. However, as already pointed out, the light ring orbit in the $\eta \neq 0$ case corresponds to a simple pole $x_{\text {pole }}(\eta)$ in the new energy function $e(x ; \eta)$. Let us define the corresponding (invariant) "velocity" $v_{\text {pole }}(\eta)$ $\equiv \sqrt{x_{\text {pole }}(\eta)}$. This motivates the introduction of the following "factored" flux function, $\hat{f}(v ; \eta)$ :

\footnotetext{
${ }^{9}$ More precisely, $e_{P_{2 n}}(x)$ is $-x$ times the Padé approximant of $-x^{-1} e_{T_{2 n}}(x)$.
} 


$$
\hat{f}(v ; \eta) \equiv\left(1-v / v_{\text {pole }}\right) \hat{\mathcal{F}}(v ; \eta),
$$

where $\quad \hat{\mathcal{F}}(v) \equiv \mathcal{F}(v) / \mathcal{F}_{N}(v)=5 \mathcal{F}(v) /\left(32 \eta^{2} v^{10}\right) \quad$ is $\quad$ the Newton-normalized flux. Note that multiplying by 1 $-v / v_{\text {pole }}$ rather than $1-\left(v / v_{\text {pole }}\right)^{2}$ has the advantage of regularizing the structure of the Taylor series of $\hat{f}(v)$ in introducing a term linear in $v$, which is absent in the flux function in Eq. (2.3) (cf. Table II). Three further inputs will allow us to construct better converging approximants to $\hat{f}(v)$. First, it is clear (if we think of $v$ as having the dimension of a velocity) that one should normalize the velocity $v$ entering the logarithms in the flux function in Eq. (2.3) to some relevant velocity scale $v_{0}$. In the absence of further information the choice $v_{0}=v_{\mathrm{LSO}}(\eta)$ seems justified (the other basic choice $v_{0}=v_{\text {pole }}$ is numerically less desirable as $v$ will never exceed $v_{\text {LSO }}$ and we wish to minimize the effect of the logarithmic terms). A second idea, to reduce the problem to a series amenable to Padé resummation, is to factorize the logarithms. This is accomplished by writing the $\hat{f}$ function in the form

$$
\hat{f}_{T_{n}}(v ; \eta)=\left(1+\sum_{k=6}^{n} \hat{l}_{k} v^{k} \ln \frac{v}{v_{\mathrm{LSO}}}\right)\left(\sum_{k=0}^{n} \hat{f}_{k} v^{k}\right),
$$

where coefficients $\hat{f}_{k}$ are $\hat{f}_{0}=1, \hat{f}_{k+1}=\hat{\mathcal{F}}_{k+1}-\hat{\mathcal{F}}_{k} / v^{\text {pole }}$ and $\hat{l}_{k}$ are constants determined from the coefficients of $\hat{\mathcal{F}}_{k}$ by relations (analogous to) Eq. (4.9) of DIS1. ${ }^{10}$ Finally, we define Padé approximants to the factored flux function $\hat{f}(v)$ as

$$
\hat{f}_{P_{n}}(v ; \eta) \equiv\left(1+\sum_{k=6}^{n} \hat{l}_{k} v^{k} \ln \frac{v}{v_{\mathrm{LSO}}^{P_{n}}(\eta)}\right) P_{m+\epsilon}^{m}\left(\sum_{k=0}^{n} \hat{f}_{k} v^{k}\right),
$$

where $v_{\mathrm{LSO}}^{P_{n}}(\eta)$ denotes the LSO velocity $\left(\equiv \sqrt{x_{\mathrm{LSO}}}\right)$ for the $v^{n}$-accurate Padé approximant of $e(x)$, and where $P_{m+\epsilon}^{m}$ denotes as before a diagonal or sub-diagonal Padé approximant with $n \equiv 2 m+\epsilon, \epsilon=0$ or 1 . The corresponding approximant of the flux $\hat{\mathcal{F}}(v)$ is then defined as

$$
\hat{\mathcal{F}}_{P_{n}}(v ; \eta) \equiv\left(1-\frac{v}{v_{\text {pole }}^{P_{n}}(\eta)}\right)^{-1} \hat{f}_{P_{n}}(v ; \eta),
$$

where $v_{\text {pole }}^{P_{n}}(\eta)$ denotes the pole velocity defined by the $v^{n}$-Padé approximant of $e(x)$. In the test mass case the exact locations of the pole and the LSO are $x_{\text {pole }}=1 / 3$ and $x_{\text {LSO }}$ $=1 / 6$, respectively (cf. Table I). We shall denote the continued fraction Padé coefficients of $\hat{f}_{P_{n}}(v)$ by $d_{k}$. They can be found in terms of $\hat{f}_{k}$ using Eqs. (2.11). At present, the most

\footnotetext{
${ }^{10}$ The variables $\hat{l}_{k}$ and $\hat{f}_{k}$ used here are equal to the variables $l_{k}$ and $f_{k}$ used in DIS1. They are "careted", here as a reminder that they represent coefficients of Newtonian-normalized quantities. The coefficients $A_{k}$ and $B_{k}$ appearing in the definition of $l_{k}$ are computed in Ref. [17].
}

accurate estimate of the flux would be $\hat{\mathcal{F}}_{P_{11}}$, defined by using the known $\eta$-dependent coefficients $\hat{f}_{k}$ for $k \leqslant 5$ and the testmass values of $\hat{f}_{k}$ and $\hat{l}_{k}$ for $k \geqslant 6$.

\section{FREQUENCY-DOMAIN PHASING FORMULAS IN THE ADIABATIC APPROXIMATION}

The time-domain $P$-approximant waveforms discussed above are computationally intensive to use in a full-scale data analysis. Recently, in DIS2 we have constructed frequency-domain representations of the $P$ approximants which are 10-50 times faster to compute than their timedomain analogues but are yet as accurate. This increases the usefulness of $P$ approximants in data analysis.

The Fourier representations are based primarily on a newly derived improved version of the stationary phase approximation appropriate to time-truncated signals. $P$ approximants cannot be modeled using the standard stationary phase approximation over the entire frequency domain. Indeed, close to the last stable orbit, where the inspiral phase terminates, one requires a modification of the stationary phase approximation. In simple terms, Ref. [6] found a way of taking into account the effect of an assumed abrupt termination of the waveform near the last stable circular orbit by introducing simple modifications to the usual stationary phase approximation. We present only the final results here; the interested reader is referred to DIS2 for details. Note that the results summarized below are quite general and can be applied to a generic chirp signal which shuts off abruptly (i.e., on a time scale $\lesssim F^{-1}$ ).

We begin with a discussion of the usual stationary phase approximation for chirp signals. Consider a signal of the form

$$
h(t)=2 a(t) \cos \phi(t)=a(t)\left[e^{-i \phi(t)}+e^{i \phi(t)}\right],
$$

where $\phi(t)$ is the implicit solution of one of the phasing formulas in Eq. (2.6), Eq. (2.7) or Eq. (2.8) for some choice of functions $E^{\prime}$ and $\mathcal{F}$ [21].

The quantity $2 \pi F(t)=d \phi(t) / d t$ defines the instantaneous GW frequency $F(t)$, and is assumed to be continuously increasing. [We assume $F(t)>0$.] Now the Fourier transform $\widetilde{h}(f)$ of $h(t)$ is defined as

$$
\begin{aligned}
\widetilde{h}(f) & \equiv \int_{-\infty}^{\infty} d t e^{2 \pi i f t} h(t) \\
& =\int_{-\infty}^{\infty} d t a(t)\left[e^{2 \pi i f t-\phi(t)}+e^{2 \pi i f t+\phi(t)}\right] .
\end{aligned}
$$

The above transform can be computed in the stationary phase approximation (SPA). For positive frequencies only the first term on the right contributes and yields the following usual SPA:

$$
\widetilde{h}^{\text {uspa }}(f)=\frac{a\left(t_{f}\right)}{\sqrt{\dot{F}\left(t_{f}\right)}} e^{i\left[\psi_{f}\left(t_{f}\right)-\pi / 4\right]}, \quad \psi_{f}(t) \equiv 2 \pi f t-\phi(t),
$$


and $t_{f}$ is the saddle point defined by solving for $t$, $d \psi_{f}(t) / d t=0$, i.e. the time $t_{f}$ when the GW frequency $F(t)$ becomes equal to the Fourier variable $f$. In the (adiabatic) approximation where Eqs. (2.1) hold, the value of $t_{f}$ is given by the following integral:

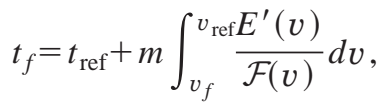

where $v_{f} \equiv(\pi m f)^{1 / 3}$. Using $t_{f}$ from the above equation and $\phi\left(t_{f}\right)$ in Eq. (2.1) one finds that

$$
\psi_{f}\left(t_{f}\right)=2 \pi f t_{\mathrm{ref}}-\phi_{\mathrm{ref}}+2 \int_{v_{f}}^{v_{\mathrm{ref}}}\left(v_{f}^{3}-v^{3}\right) \frac{E^{\prime}(v)}{\mathcal{F}(v)} d v .
$$

The big computational advantage of Eq. (3.5) [with respect to its time-domain counterpart, Eq. (2.1)] is that, in the frequency domain, there are no equations to solve iteratively; the Fourier amplitudes are given as explicit functions of frequency.

In the Fourier domain too there are many inequivalent ways in which the phasing $\psi_{f}$ can be worked out. Here we mention only the most popular:

(f1) Substitute (without doing any re-expansion or resummation) for the energy and flux functions their PN expansions or the $P$ approximants of energy and flux functions and solve the integral in Eq. (3.5) numerically to obtain the $T$ approximant SPA or $P$ approximant SPA, respectively.

(f2) Use PN expansions of energy and flux but re-expand the ratio $E^{\prime}(v) / \mathcal{F}(v)$ in Eq. (3.5) in which case the integral can be solved explicitly. This leads to the following explicit, Taylor-like, Fourier domain phasing formula:

$$
\psi_{f}\left(t_{f}\right)=2 \pi f t_{\mathrm{ref}}-\phi_{\mathrm{ref}}+\tau_{N} \sum_{k=0}^{5} \hat{\tau}_{k}(\pi m f)^{(k-5) / 3}
$$

where $\hat{\tau}_{k}$ are the chirp parameters listed in Table II. Equation (3.6) is one of the standardly used frequency-domain phasing formulas. Therefore, we shall use that as one of the models in our comparison of different inspiral model waveforms. We refer to it as "type-f2" frequency-domain phasing.

Just as in the time-domain, the frequency-domain phasing is most efficiently computed by a pair of coupled, non-linear, ODEs:

$$
\frac{d \psi}{d f}-2 \pi t=0, \quad \frac{d t}{d f}+\frac{\pi m^{2}}{3 v^{2}} \frac{E^{\prime}(f)}{\mathcal{F}(f)}=0
$$

rather than by numerically computing the integral in Eqs. (3.5).

Next we correct the performance of the usual SPA by including edge corrections arising as a consequence of modeling the time-domain signal as being abruptly terminated at a time $t=t_{\max }$ (time-truncated chirp) when the GW frequency reaches $F=F_{\max }$. In practice, we expect that $F_{\max }$ will be of order the GW frequency at the LSO. However, we prefer to leave unspecified the exact value of $F_{\max }$. The idea is to use $F_{\text {max }}$ as a free model parameter, to be varied so as to maximize the overlap (see Sec. V) between the $t_{\text {max }}$-truncated template and the real signal. Such timetruncated signals can be represented as

$$
h(t)=2 a(t) \cos \phi(t) \theta\left(t_{\max }-t\right)
$$

where $\theta$ denotes the Heaviside step function, i.e. $\theta(x)=0$ if $x<0$ and $\theta(x)=1$ when $x \geqslant 0$. The effect of this time windowing has been modeled in DIS2 and the result is that the Fourier transform of such a time-truncated signal can be accurately represented in the two regions $f \leqslant F_{\max }$ and $f$ $\geqslant F_{\text {max }}$, by

$$
\begin{aligned}
f \leqslant F_{\text {max }}: \tilde{h}_{<}^{\text {ispa }}(f)=\mathcal{C}\left(\zeta_{<}(f)\right) \frac{a\left(t_{f}\right)}{\sqrt{\dot{F}\left(t_{f}\right)}} e^{i\left[\psi_{f}\left(t_{f}\right)-\pi / 4\right]}, \\
f \geqslant F_{\text {max }}: \widetilde{h}_{>}^{\text {ispa }}(f)=\mathcal{C}\left(\zeta_{>}(f)\right) \frac{a\left(t_{\text {max }}\right)}{\sqrt{\dot{F}\left(t_{\text {max }}\right)}} \\
\quad \times \exp \left[i \psi_{f}\left(t_{\text {max }}\right)+i \frac{\pi\left(f-F_{\text {max }}\right)^{2}}{\dot{F}\left(t_{\text {max }}\right)}-i \pi / 4\right],
\end{aligned}
$$

where the label "ispa" stands for improved SPA, $\mathcal{C}$ is essentially the complementary error function, $2 \mathcal{C}(\zeta)$ $\equiv \operatorname{erfc}\left(e^{i \pi / 4} \zeta\right)$, and $\zeta$ is computed using

$$
\begin{aligned}
f<F_{\max }: \zeta_{<} & \equiv-\left[\psi_{f}\left(t_{f}\right)-\psi_{f}\left(t_{\max }\right)\right]^{1 / 2}, \\
f \geqslant F_{\max }: \zeta_{>}(f) & =\frac{\sqrt{\pi}\left(f-F_{\max }\right)}{\sqrt{\dot{F}\left(t_{\max }\right)}} .
\end{aligned}
$$

The error function needed in calculating $\mathcal{C}(f)$ may be numerically computed using the NAG [22] library S15DDF. Note the denominator $\sqrt{\dot{F}\left(t_{\max }\right)}$ entering Eqs. (3.9) and (3.10). We define a generic time-truncated signal as a chirp for which this denominator is finite (neither infinite nor zero). [These signals were called "Newtonian-like", in Ref. [6]. We do not keep this name here to emphasize that the results, Eqs. (3.9) and (3.10), apply also to relativistic models, as long as $\dot{F}\left(t_{\max }\right)$ is finite.]

The exceptional (non-generic) case where $\sqrt{\dot{F}\left(t_{\text {max }}\right)}$ becomes infinite arises if one tries to keep using the simple adiabatic phasing approximation up to the last stable orbit defined by the corresponding (approximate) energy function $E(v)$. This exceptional case can also be dealt with at the price of a more complicated modification of the usual stationary phase result (see Ref. [6] for details). We do not enter into details here because the recent work [7] on the transition between the adiabatic inspiral and the plunge has shown that the adiabatic approximation breaks down just before the LSO, and that $\dot{F}(t)$ never becomes infinite. 


\section{TIME-DOMAIN PHASING FORMULA BEYOND THE ADIABATIC APPROXIMATION}

In the above, we restricted ourselves to the standard "adiabatic approximation," where one estimates the phasing by combining the energy-balance equation $d E_{\text {tot }} / d t=-\mathcal{F}$ with some resummed estimates for the energy and flux as functions of the instantaneous circular orbital frequency. Recently, Buonnano and Damour [7] have introduced a new approach to GWs from coalescing binaries which is no longer limited to the adiabatic approximation, and which is expected to describe rather accurately the transition between the inspiral and the plunge, and to give also a first estimate of the following plunge signal. The approach of [7] is essentially, like [5,6], a re-summation technique which consists of two main ingredients: (i) the "conservative" (damping-free) part of the dynamics (effectively equivalent to the specification of the $E(v)$ in the previous approaches) is resummed by a new technique which replaces the two-body dynamics by an effective one-body dynamics [7] and (ii) the "damping", part of the dynamics [equivalent to the specification of the $\mathcal{F}(v)]$ is constructed by borrowing the re-summation technique introduced in [5]. In practical terms, the time-domain waveform is obtained as the following function of the reduced time $\hat{t}=t / m$ :

$h(\hat{t})=\mathcal{C} v_{\omega}^{2}(\hat{t}) \cos \left[\phi_{\mathrm{GW}}(\hat{t})\right], \quad v_{\omega} \equiv\left(\frac{d \varphi}{d \hat{t}}\right)^{1 / 3}, \quad \phi_{\mathrm{GW}} \equiv 2 \varphi$.

The orbital phase $\varphi(\hat{t})$ entering Eq. (4.1) is given by integrating a system of ODEs:

$$
\begin{aligned}
\frac{d r}{d \hat{t}} & =\frac{\partial \hat{H}}{\partial p_{r}}\left(r, p_{r}, p_{\varphi}\right), \\
\frac{d \varphi}{d \hat{t}} & =\hat{\omega} \equiv \frac{\partial \hat{H}}{\partial p_{\varphi}}\left(r, p_{r}, p_{\varphi}\right), \\
\frac{d p_{r}}{d \hat{t}}+\frac{\partial \hat{H}}{\partial r}\left(r, p_{r}, p_{\varphi}\right) & =0, \\
\frac{d p_{\varphi}}{d \hat{t}} & =\hat{\mathcal{F}}_{\varphi}\left(\hat{\omega}\left(r, p_{r}, p_{\varphi}\right)\right) .
\end{aligned}
$$

The reduced Hamiltonian $\hat{H}$ (of the associated one-body problem) is given, at the $2 \mathrm{PN}$ approximation, by

$$
\begin{aligned}
& \hat{H}\left(r, p_{r}, p_{\varphi}\right) \\
& \quad=\frac{1}{\eta} \sqrt{1+2 \eta\left[\sqrt{A(r)\left(1+\frac{p_{r}^{2}}{B(r)}+\frac{p_{\varphi}^{2}}{r^{2}}\right)}-1\right]},
\end{aligned}
$$

where

$$
A(r) \equiv 1-\frac{2}{r}+\frac{2 \eta}{r^{3}}, \quad B(r) \equiv \frac{1}{A(r)}\left(1-\frac{6 \eta}{r^{2}}\right)
$$

The 3PN version of $\hat{H}$ has been recently obtained [2]. The damping force $\mathcal{F}_{\varphi}$ is approximated by

$$
\hat{\mathcal{F}}_{\varphi}=-\frac{1}{\eta v_{\omega}^{3}} \mathcal{F}_{P_{n}}\left(v_{\omega}\right)
$$

where $\mathcal{F}_{P_{n}}\left(v_{\omega}\right)=\frac{32}{5} \eta^{2} v_{\omega}^{10} \hat{\mathcal{F}}_{P_{n}}\left(v_{\omega}\right)$ is the flux function used in $P$ approximants discussed above.

The system, Eq. (4.2), allows one to describe the smooth transition which takes place between the inspiral and the plunge [while the system (2.2) becomes spuriously singular at the LSO, where $\left.E^{\prime}\left(v_{\text {LSO }}\right)=0\right]$. Reference [7] advocated to continue using Eqs. (4.2) after the transition, to describe the waveform emitted during the plunge and to match around the "light ring"' to a "'merger" waveform, described, in the first approximation, by the ringing of the least-damped quasinormal mode of a Kerr black hole [see Eq. (6.2) of [7]]. This technique is the most complete which is available at present. It includes (in the best available approximation and for nonspinning black holes) most of the correct physics of the problem, and leads to a specific prediction for the complete waveform (inspiral + plunge + merger) emitted by coalescing binaries. Because of its completeness, we shall use it as our "fiducial exact", waveform in our comparison between different search templates.

The initial data needed in computing this effective onebody waveform are as follows: In gravitational wave data analysis we are normally given an initial frequency $f_{0}\left(\hat{\omega}_{0}\right.$ $\equiv \pi m f_{0}$ ) corresponding to the lower cutoff of a detector's sensitivity window, at which to begin the waveform. The initial phase of the signal will not be known in advance but in order to gauge the optimal performance of our approximate templates we maximize the overlap (see Sec. V) over the initial phases of both the fiducial exact signal (i.e., the effective-one-body waveform) and the approximate template. The general analytical result of this maximization was discussed in Appendix B of DIS1. In the terminology of this appendix, these fully phase-maximized overlaps were called the best overlaps [they are given by Eq. (B.11) of DIS1]. As discussed in Appendix B of DIS1, there are two distinct measures of the closeness of two signals: the best overlap (maximized over the phases of both the template and the exact signal) and the minimax overlap (maximized over the template phase, with the worst possible exact phase). In an investigation such as ours (where we are interested in the optimal mathematical closeness between different signals), the best overlap is the mathematically cleanest measure of closeness of two families of templates, and we shall use it here. In addition, we shall also maximize over the other template parameters (in particular, the masses) to get an intrinsic measure of the closeness of two families of templates. Note that 
the resulting fully maximized overlaps are different from the maximized ambiguity function of Ref. [23] and the fitting factor of Ref. [24]. The latter (identical, but given different names by different authors) quantities are well-defined measures of the closeness of two signals only within the (simplifying) approximation where signals in quadrature are orthogonal. This is, however, not the case for the signals we consider, and at the accuracy at which we are working.

For the computation of the best overlaps, it is sufficient to construct two signal waveforms and two template waveforms, one with phase equal to 0 and another with phase equal to $\pi / 2$. The rest of the initial data $\left(r_{0}, p_{r}^{0}, p_{\varphi}^{0}\right)$ are found using

$$
\begin{aligned}
& r_{0}^{3}\left[\frac{1+2 \eta\left(\sqrt{z\left(r_{0}\right)}-1\right)}{1-3 \eta / r_{0}^{2}}\right]-\hat{\omega}_{0}^{-2} \\
& \quad=0 \\
& p_{\varphi}^{0}=\left[\frac{r_{0}^{2}-3 \eta}{r_{0}^{3}-3 r_{0}^{2}+5 \eta}\right]^{1 / 2}, \quad p_{r}^{0}=\frac{\mathcal{F}_{\varphi}(\hat{\omega})}{C\left(r_{0}, p_{\varphi}^{0}\right)\left(d p_{\varphi}^{0} / d r_{0}\right)}
\end{aligned}
$$

where $z(r)$ and $C\left(r, p_{\varphi}\right)$ are given by

$$
\begin{aligned}
z(r) & =\frac{r^{3} A^{2}(r)}{r^{3}-3 r^{2}+5 \eta}, \\
C\left(r, p_{\varphi}\right) & =\frac{1}{\eta \hat{H}\left(r, 0, p_{\varphi}\right) \sqrt{z(r)}} \frac{A^{2}(r)}{\left(1-6 \eta / r^{2}\right)} .
\end{aligned}
$$

The plunge waveform is terminated when the radial coordinate attains the value at the light ring $r_{\text {lr }}$ given by the solution to the equation,

$$
r_{\mathrm{lr}}^{3}-3 r_{\mathrm{lr}}^{2}+5 \eta=0
$$

The subsequent "merger" waveform is constructed as in Ref. [7].

\section{RESULTS AND CONCLUSIONS}

In this section we compare the performances of various signal models by choosing as fiducial "exact" signal model the effective one-body waveforms discussed in the previous section. An important yardstick for comparing different waveforms is the overlap: Given two waveforms $h$ and $g$ their overlap is defined as

$$
\mathcal{O}(h, g)=\frac{\langle h, g\rangle}{\langle h, h\rangle^{1 / 2}\langle g, g\rangle^{1 / 2}} .
$$

In the above equation the scalar product $\langle$,$\rangle is defined as$

$$
\langle h, g\rangle=2 \int_{0}^{\infty} \frac{d f}{S_{h}(f)} \widetilde{h}(f) \tilde{g}^{*}(f)+\text { c.c. }
$$

where c.c. denotes complex conjugation and $S_{h}(f)$ is the one-sided detector noise spectral density $\left[S_{h}^{\text {one-sided }}\right.$ $=2 S_{h}^{\text {two-sided }}$ leading to the factor of 2 in Eq. (5.2), compared to the definition used in [6], where we always use the twosided noise]. See the Appendix below for the noise performances of the various detectors.

First, in Fig. 1 we compare the signal-to-noise ratios (SNRs), expected in GEO, LIGO and VIRGO, for equal mass binaries located at $100 \mathrm{Mpc}$, when detecting an "exact" signal $h$ by means of a bank of templates $k$ :

$$
\rho \equiv \frac{S}{\bar{N}}=\frac{|\langle k, h\rangle|}{\langle k, k\rangle^{1 / 2}}=|\mathcal{O}(k, h)|\langle h, h\rangle^{1 / 2} .
$$

Thick lines plot the SNR obtained when $k=h$, i.e. when the template perfectly matches our fiducial exact waveform (i.e. the effective one-body waveform including its "ringing tail"), and thin lines show how that gets degraded when we use for $k$ the best post-Newtonian template $T^{f 2}$ [cf. Eq. (3.6)] truncated at the test-mass $\mathrm{LSO}, F_{\mathrm{LSO}}^{\mathrm{GW}}=4400 M_{\odot} / m \mathrm{~Hz}$, assuming still that the true signal $h$ is the one-body effective waveform. (As usual, see, e.g., Sec. IV A of [6], where we averaged over all the angles.) The overlaps $\mathcal{O}(k, h)$ are maximized over the time lag and the two phases (as explained in the previous section), as well as over the two individual masses $m_{1}$ and $m_{2}{ }^{11}$ The greater SNR achieved by effective one-body waveforms for higher masses, as compared to Fig. 1 of DIS2, is due to the plunge phase present in these waveforms. We have checked that the final merger signal, modeled as a quasi-normal mode, has a numerically insignificant effect in both the SNR and the overlaps: the overlap between our fiducial exact waveform and an effective one-body waveform minus quasi-normal modes is greater than 0.98 for double black holes of masses smaller than $(40,40) M_{\odot}$.

The origin of the enhancement in SNR is easily understood. The plunge waveform begins around $f \simeq F_{\text {LSO }}$ and lasts until $f \simeq 2 F_{\text {LSO }}$ [7]. The mass of a binary whose last stable orbit velocity is equal to the characteristic velocity $v_{p}$ of LIGO's peak sensitivity is $m=34.8 M_{\odot}$. Therefore, effective one-body waveforms from binaries of masses in the range $35 M_{\odot} \lesssim m \leqq 70 M_{\odot}$ have larger SNRs for LIGO, than the usual SPA models. It is, therefore, crucial to go beyond the waveforms given in the adiabatic approximation to take advantage of these higher SNRs for larger masses. Indeed, the SNRs being as high as 8 , for one detector and a source at $100 \mathrm{Mpc}$, a network of four detectors (two LIGOs, GEO and VIRGO) will be able to reliably search for such systems as far as $150-200 \mathrm{Mpc}$.

Next, in Table III we show the fully maximized overlaps of effective one-body waveforms with signal models $T^{\text {t1 }}$ (column 2), $T^{\mathrm{t} 3}$ (column 3), $T^{\mathrm{f} 1}$ (column 4), $T^{\mathrm{f} 2}$ (column 5) and $P$ (column 6 ) for four typical binaries. The time-domain

\footnotetext{
${ }^{11}$ The plots are jagged because we have computed the SNR numerically by first generating the waveform in the time domain and then using its discrete Fourier transform in Eq. (5.3).
} 
TABLE III. Fully maximized overlaps of the fiducial exact $(X)$ waveform (effective-one-body signal [7]) with (1) the standard time-domain post-Newtonian approximations of type $\mathrm{t} 1$ and $\mathrm{t} 3\left(T^{\mathrm{t} 1}, T^{\mathrm{t} 3}\right)$, given in Eq. (2.6) and Eq. (2.8) and Table II, (2) the frequency-domain usual stationary phase approximations of type 1 and $2\left(T^{\mathrm{f} 1}, T^{\mathrm{f} 2}\right)$, given in Eqs. (3.5), (3.3) and (3.6) and Table II, and (3) the time-domain $P$ approximants $(P)$ - energy function as given by Eqs. (2.14) and Eqs. (2.16), flux function in Eqs. (2.20) and (2.21), and coefficients enlisted in Tables I and II. The overlaps, which are computed using the LIGO noise curve, are maximized not only over the time lag and the initial phases of both the fiducial exact signal and the approximate template (by using two signal and two template waveforms, with phases equal to 0 and $\pi / 2$ [5]), but also over the two masses $m_{1}$ and $m_{2}$ of the approximate signal models. (The optimal masses are given below the overlaps.) The time-domain $T^{\mathrm{t} 3}$ approximants are terminated when $\dot{F}=0$ and the other signals are terminated when $F(t)=f_{\text {LSO }}$, the LSO frequency being determined consistently using $E_{A}^{\prime}(v)=0$ where $E_{A}(v)$ is the corresponding approximate energy function.

\begin{tabular}{|c|c|c|c|c|c|}
\hline$k$ & $\left\langle X, T_{k}^{\mathrm{t} 1}\right\rangle$ & $\left\langle X, T_{k}^{\mathrm{t} 3}\right\rangle$ & $\left\langle X, T_{k}^{\mathrm{f} 1}\right\rangle$ & $\left\langle X, T_{k}^{\mathrm{f} 2}\right\rangle$ & $\left\langle X, P_{k}\right\rangle$ \\
\hline & \multicolumn{5}{|c|}{$m_{1}=m_{2}=15 M_{\odot}$} \\
\hline \multirow[t]{2}{*}{4} & 0.8881 & 0.9488 & 0.8644 & 0.8144 & 0.8928 \\
\hline & $(15.2,14.1)$ & $(16.3,16.4)$ & $(14.7,14.9)$ & $(16.0,16.1)$ & $(14.7,15.1)$ \\
\hline \multirow[t]{3}{*}{5} & 0.8794 & 0.8479 & 0.7808 & 0.8602 & 0.8929 \\
\hline & $(17.3,16.4)$ & $(17.6,17.9)$ & $(16.8,16.7)$ & $(15.2,14.4)$ & $(15.4,14.3)$ \\
\hline & \multicolumn{5}{|c|}{$m_{1}=m_{2}=10 M_{\odot}$} \\
\hline \multirow[t]{2}{*}{4} & 0.9604 & 0.9298 & 0.9581 & 0.9109 & 0.9616 \\
\hline & $(10.1,9.6)$ & $(10.5,10.3)$ & $(10.0,9.7)$ & $(10.5,10.6)$ & $(10.0,10.2)$ \\
\hline \multirow[t]{3}{*}{5} & 0.8814 & 0.8490 & 0.8616 & 0.9529 & 0.9610 \\
\hline & $(11.4,10.6)$ & $(11.4,11.7)$ & $(10.7,11.0)$ & $(10.3,9.7)$ & $(10.4,9.7)$ \\
\hline & \multicolumn{5}{|c|}{$m_{1}=10 M_{\odot}, m_{2}=1.4 M_{\odot}$} \\
\hline \multirow[t]{2}{*}{4} & 0.9847 & 0.9673 & 0.9835 & 0.9721 & 0.9937 \\
\hline & $(1.27,11.1)$ & $(0.95,16.6)$ & $(1.27,11.1)$ & $(0.96,16.4)$ & $(1.35,10.5)$ \\
\hline \multirow[t]{3}{*}{5} & 0.9452 & 0.6811 & 0.9394 & 0.9922 & 0.9941 \\
\hline & $(0.82,20.4)$ & $(1.11,15.7)$ & $(0.82,20.4)$ & $(1.34,10.5)$ & $(1.37,10.2)$ \\
\hline & \multicolumn{5}{|c|}{$m_{1}=m_{2}=1.4 M_{\odot}$} \\
\hline \multirow[t]{2}{*}{4} & 0.8828 & 0.8538 & 0.8830 & 0.8503 & 0.9719 \\
\hline & $(1.40,1.39)$ & $(1.42,1.39)$ & $(1.40,1.39)$ & $(1.44,1.37)$ & $(1.47,1.34)$ \\
\hline \multirow[t]{2}{*}{5} & 0.8522 & 0.7643 & 0.8522 & 0.9994 & 0.9945 \\
\hline & $(1.46,1.35)$ & $(1.43,1.38)$ & $(1.46,1.35)$ & $(1.45,1.35)$ & $(1.49,1.32)$ \\
\hline
\end{tabular}

$T^{\mathrm{t} 3}$ approximants are terminated when $\dot{F}=0$ and the other approximants are terminated when $F(t)=f_{\mathrm{LSO}}$, the LSO being determined consistently using $E_{A}^{\prime}(v)=0$, where $E_{A}(v)$ is the corresponding approximate energy function. The overlaps are computed with the (initial) LIGO noise curve given in the Appendix.

In addition to maximization over the time lags and the phases as explained earlier, we also maximize over the masses of the approximate waveforms, keeping the masses of the exact waveforms fixed. We note that none of the models have good overlaps with the "exact" one for heavier mass binaries. This is as expected since it is for heavier masses that the characteristic plunge phase makes a significant difference between the approximate and the exact models. The relative performances of the $2 \mathrm{PN}$ and 2.5PN Taylor templates depends on the choice of the scheme used as is evident from columns 3 and 4 in Table III. This is consistent with the results of Ref. [25]. (The numerical results must not be compared since they quote values for the "advanced", LIGO.) Some Taylor models are "effectual" (large maximized overlaps), but at the cost of high biases in the parameters (i.e. in the terminology of [5] they are not "faithful"), For example in the case of a fiducial exact $(1.4,10) M_{\odot}$ system $T_{5}^{t 1}$ reaches 0.9452 for mass values $(0.8,20) M_{\odot}$. Thus $T$ approximants in the time domain are significantly inferior to the other models, both for their erratic convergence properties (the $v^{5}$-accurate templates being worse than the $v^{4}$-accurate ones) and for their poor parameter estimation performance.

To further explore the performance of the various models we plot in Fig. 2 their intrinsic frequency evolutions in the LIGO band; i.e., we plot $\dot{F} / F^{2}$ versus $F$. The plot corresponds, in the fiducial exact case, to a binary black hole of $(10,10) M_{\odot}$. For the approximate models we exhibit the frequency evolution of the system that achieves the maximum overlap. As expected, the maximum overlap is obtained for 


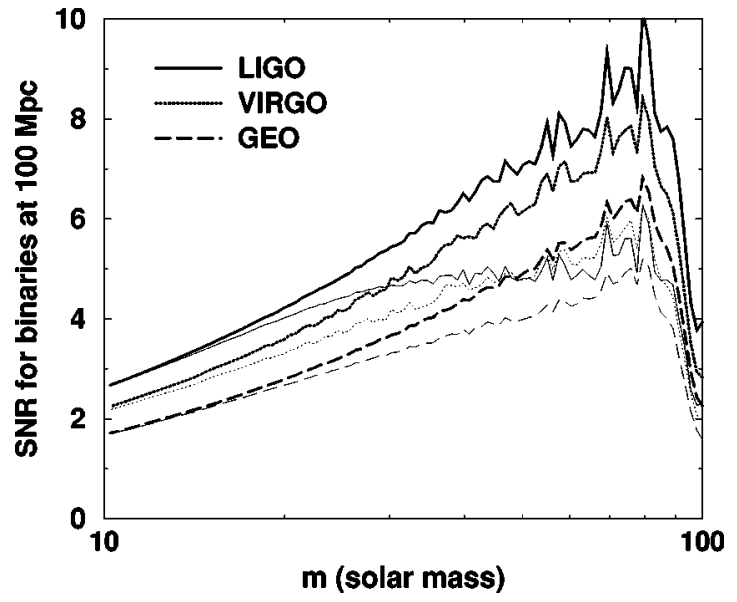

FIG. 1. Signal-to-noise ratios in GEO, LIGO-I and VIRGO when using as Fourier-domain template the post-Newtonian model Eq. (3.6) $\left(T^{f 2}\right)$, truncated at the test mass $F_{\mathrm{LSO}}=4400 M_{\odot} / m \mathrm{~Hz}$ (thin lines), compared to the optimal one obtained when the template coincides with the fiducial "exact" (effective one-body) signal (thick lines). As usual, we averaged over all the angles. The overlaps are maximized over the time lags, the phases, and the two individual masses $m_{1}$ and $m_{2}$. The plots are jagged because we have computed the SNR numerically by first generating the fiducial "exact" waveform in the time domain and then using its discrete Fourier transform in Eq. (5.3). The greater SNR achieved by effective one-body waveforms for higher masses, as compared to Fig. 1 of DIS2, is due to the plunge phase present in these waveforms. Observe that the presence of the plunge phase in the latter significantly (up to a factor of 1.5) increases the SNR for masses $m$ $>35 M_{\odot}$. Using the effective one-body templates will, therefore, enhance the search volume of the interferometric network by a factor of 3 or 4
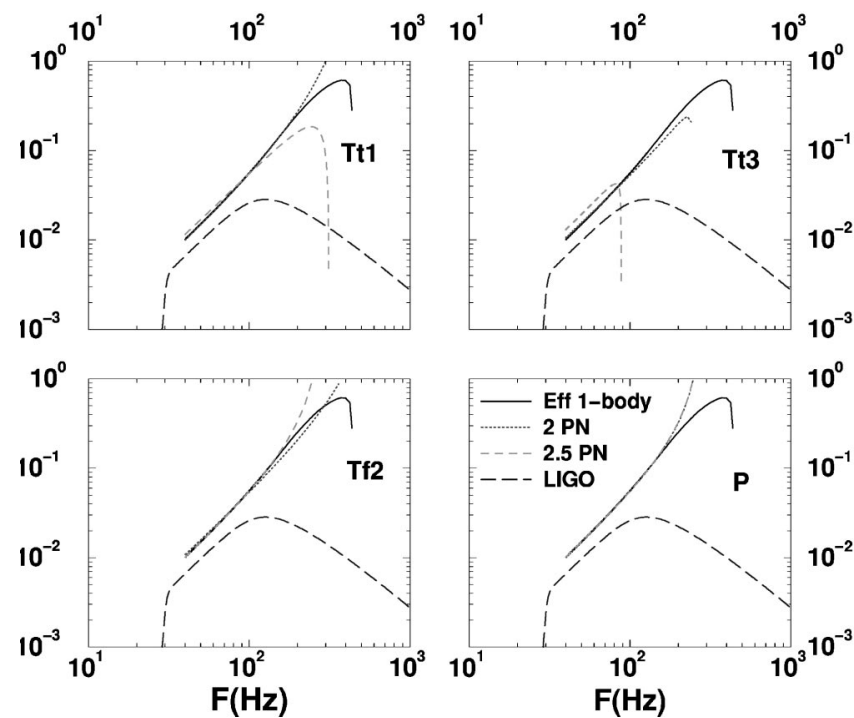

FIG. 2. The frequency evolution of the various approximate models is compared with the fiducial exact $(10,10) M_{\odot}$ model in the LIGO band. To indicate the effect on the overlap, we also plot the weighting function $1 / h_{n}(f)$ for initial LIGO (not to scale), which is a measure of the detector's sensitivity.

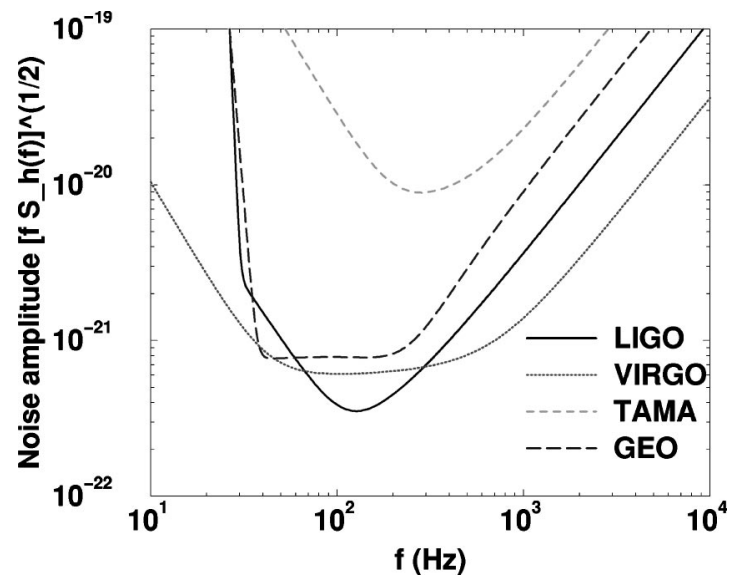

FIG. 3. The effective noise $h_{n}=\sqrt{f S_{h}(f)}$ in various groundbased interferometers.

template parameters such that the intrinsic frequency evolution of the template waveform is "tangent" to the exact one, near the maximum sensitivity of the detector. This can always be achieved by fitting the mass parameters. The question is whether such a local "tangency" ensures a sufficiently good "global" agreement. For instance, we note that the $T^{\mathrm{t1}, 3} 2.5 \mathrm{PN}$ models fare poorly in globally mimicking the frequency evolution of the exact waveform. This is consistent with their returning the worst overlaps of all. On the other hand, even though the $T^{f 2}$ models do not reproduce the exact model over as large a range as the $P$ approximants, they achieve nearly as large overlaps as the $P$ approximants, because they can be made (by optimizing the masses) to agree well with the exact model over most of the sensitive part of the LIGO band. The $P$ approximants are able to mimic the "exact" evolution the best with little bias in the masses but, being based on the adiabatic approximation, they fail to capture the smooth transition to plunge. ${ }^{12}$ The filters using the effective one-body approach go beyond the adiabatic approximation and include a smooth transition to plunge and merger. They, therefore, supersede the adiabaticlimited $P$ approximants. This difference between the two resummed versions of binary signal models is important for masses larger than about $20 M_{\odot}$.

Note that, following [7], we have generated the effectiveone-body model using the adimensional time $\hat{t}=t / \mathrm{m}$. (This trivially extends beyond the $2 \mathrm{PN}$ approximation [2].) It has been recently emphasized [26] (in the context of the $T$ and $P$ approximants, where one can also simplify formulas by working with $\hat{t}=t / \mathrm{m}$ ) that there are many computational advantages in working with such adimensionalized time models. Indeed, the phase evolution becomes completely independent of the total mass of the system. This, together with the fact that the evolution can be computed from a system of

\footnotetext{
${ }^{12} \mathrm{We}$ have confirmed that the $P$ approximants return the best overlaps when truncated at the $P$-defined LSO. Maximizing over a cutoff frequency smaller than the $P$-defined $F_{\text {LSO }}$ (which turns out to be higher than the effective-one-body-defined one) does not improve the overlaps.
} 
TABLE IV. One-sided noise power spectral densities (PSDs) of initial interferometers, $S_{h}(f)$. For each detector the noise PSD is given in terms of a dimensionless frequency $x=f / f_{0}$ and rises steeply above a lower cutoff $f_{s}$.

\begin{tabular}{lccc}
\hline \hline Detector & $f_{s} / \mathrm{Hz}$ & $f_{0} / \mathrm{Hz}$ & $10^{46} \times S_{h}(x) / \mathrm{Hz}^{-1}$ \\
\hline GEO & 40 & 150 & {$\left[(3.4 x)^{-30}+34 x^{-1}+20\left(1-x^{2}+x^{4} / 2\right) /\left(1+x^{2} / 2\right)\right]$} \\
LIGO-I & 40 & 150 & $9.00\left[(4.49 x)^{-56}+0.16 x^{-4.52}+0.52+0.32 x^{2}\right]$ \\
TAMA & 75 & 400 & $75\left[x^{-5}+13 x^{-1}+9\left(1+x^{2}\right)\right]$ \\
VIRGO & 20 & 500 & $3.24\left[(6.23 x)^{-5}+2 x^{-1}+1+x^{2}\right]$ \\
\hline \hline
\end{tabular}

ODEs, makes the computation and storage of templates required in a search for binary black holes and neutron stars in interferometer data computationally inexpensive as compared to the conventional method that uses a 2-dimensional lattice of templates.

We are currently estimating the effects of unknown parameters in the 3PN motion and wave generation [27]. The extension of the type of work presented here to go beyond the restricted post-Newtonian approach and also to include the effects due to spin and eccentricity, needs to be systematically investigated.

To conclude, we believe that many of the new technical tools developed in [5,6] and briefly summarized above are useful ingredients for constructing effectual and fastcomputed inspiral templates. For example, (i) the specific Padé-based resummation of the GW flux introduced in [5] is an important ingredient of the construction in [7] of an accurate non-adiabatic waveform and (ii) the improved SPA technique derived in [6] could be used to derive analytical approximations to the frequency-domain version of these effective-one-body waveforms. In view of our ignorance of the "exact'" waveform emitted near and after the LSO crossing, the best strategy is probably to construct a bank of templates which cover a large range of possibilities with special weight being given to the templates incorporating the best tested re-summation methods (such as $P$ approximants, and the effective-one-body approach [7]). Because of the admittedly quantitatively rough, but plausibly qualitatively correct, description of the plunge signal given by the EOB approach, we also recommend to include some sort of multi-parameter template which qualitatively looks like Fig. 12 of [7], but which introduces some flexibility both in the phasing evolution during the plunge and in the location of the matching to the ring-down (with the possible inclusion of several quasinormal modes). Finally, we emphasize the importance of modelling the transition to the plunge and of including the signal emitted during the plunge: this leads to a very significant enhancement of the signal-to-noise ratio, from about 4.5 to 8 , for a source at $100 \mathrm{Mpc}$.

\section{ACKNOWLEDGMENTS}

The GEO noise curve is based on data provided by G. Cagnoli and J. Hough, LIGO is a fit to the envelope of the data from K. Blackburn, TAMA is from M.-K. Fujimoto and VIRGO is from J-Y. Vinet. We thank Alessandra Buonnano for sharing her MATHEMATICA codes for waveform generation with us. B.R.I. and B.S.S. would like to thank IHES, France, and AEI, Germany, for hospitality during the final stages of this work.

\section{APPENDIX: NOISE POWER SPECTRUM OF INITIAL INTERFEROMETERS}

In this short appendix we list the expected one-sided noise power spectral densities of the various ground-based interferometers (Table IV) and plot the effective noise ${ }^{13} h_{n}$ $\equiv \sqrt{f S_{h}(f)}$ in Fig. 3 .

\footnotetext{
${ }^{13}$ Our effective noise $h_{n}$ is the same as what has been conventionally called $h_{\mathrm{rms}}$ in the literature.
}

[1] P. Jaranowski and G. Schäfer, Phys. Rev. D 57, 5948 (1998); 57, 7274 (1998); 60, 124003 (1999).

[2] T. Damour, P. Jaranowski, and G. Schäfer, Phys. Rev. D 62, 021501 (2000); 62, 044024 (2000); 62, 084011 (2000).

[3] L. Blanchet and G. Faye, Phys. Lett. A 271, 58 (2000); L. Blanchet and G. Faye, gr-qc/0004009; gr-qc/0006100; gr-qc/0007051.

[4] C. Cutler et al., Phys. Rev. Lett. 70, 2984 (1993).

[5] T. Damour, B.R. Iyer, and B.S. Sathyaprakash, Phys. Rev. D 57, 885 (1998); referred to as DIS1 in this paper.

[6] T. Damour, B.R. Iyer, and B.S. Sathyaprakash, Phys. Rev. D 62, 084036 (2000), referred to as DIS2 in this paper.
[7] A. Buonanno and T. Damour, Phys. Rev. D 62, 064015 (2000).

[8] A. Buonanno and T. Damour, Phys. Rev. D 59, 084006 (1999).

[9] E.E. Flanagan and S.A. Hughes, Phys. Rev. D 57, 4535 (1998); 57, 4566 (1998).

[10] V.M. Lipunov, K.A. Postnov, and M.E. Prokhorov, New Astron. 2, 43 (1997); S.P. Zwart and S. McMillan, "Black hole mergers in the Universe,' astro-ph/9910061.

[11] A. Buonanno and T. Damour, "Binary black holes coalescence: transition from adiabatic inspiral to plunge,' contributed paper to the IX Marcel Grossmann Meeting in Rome, 2000, gr-qc/0011052. 
[12] J. Baker, B. Brugmann, M. Campanelli, and C.O. Lousto, Class. Quantum Grav. 17, L149 (2000).

[13] T. Damour and N. Deruelle, Phys. Lett. 87A, 81 (1981); C. R. Seances Acad. Sci., Ser. 2 293, 537 (1981); T. Damour, ibid. 294, 1355 (1982); in Gravitational Radiation, edited by N. Deruelle and T. Piran (North-Holland, Amsterdam, 1983), pp. 59-144.

[14] L. Blanchet, T. Damour, B.R. Iyer, C.M. Will, and A.G. Wiseman, Phys. Rev. Lett. 74, 3515 (1995); L. Blanchet, T. Damour, and B.R. Iyer, Phys. Rev. D 51, 5360 (1995); C.M. Will and A.G. Wiseman, ibid. 54, 4813 (1996); L. Blanchet, B.R. Iyer, C.M. Will, and A.G. Wiseman, Class Quantum Grav. 13, 575 (1996).

[15] L. Blanchet, Phys. Rev. D 54, 1417 (1996).

[16] E. Poisson, Phys. Rev. D 52, 5719 (1995).

[17] T. Tanaka, H. Tagoshi, and M. Sasaki, Prog. Theor. Phys. 96, 1087 (1996).

[18] E. Poisson, Phys. Rev. D 47, 1497 (1993).

[19] C. Cutler, L.S. Finn, E. Poisson, and G.J. Sussmann, Phys.
Rev. D 47, 1511 (1993).

[20] C.M. Bender and S.A. Orszag, Advanced Mathematical Methods for Scientists and Engineers (McGraw Hill, Singapore, 1984).

[21] At the Newtonian order, in terms of chirp mass, $\mathcal{M}=\eta^{3 / 5} \mathrm{~m}$, the chirp amplitude $a(t)=\mathcal{C}_{\mathcal{M}}[\pi \mathcal{M} F(t)]^{2 / 3}$, phase $\phi(t)=\phi_{c}$ $-2\left[\left(t_{c}-t\right) / 5 \mathcal{M}\right]^{5 / 8}$, and frequency of the gravitational waves $\pi \mathcal{M} F(t)=\left[5 \mathcal{M} / 256\left(t_{c}-t\right)\right]^{3 / 8}$.

[22] The Numerical Algorithms Group Limited, Oxford, United Kingdom.

[23] B.S. Sathyaprakash and S.V. Dhurandhar, Phys. Rev. D 44, 3819 (1991).

[24] T. Apostolatos, Phys. Rev. D 52, 605 (1995).

[25] W. Tichy, E.E. Flanagan, and E. Poisson, Phys. Rev. D 61, 104015 (2000).

[26] B.S. Sathyaprakash, Class. Quantum Grav. 17, L157 (2000),

[27] T. Damour, B.R. Iyer, P. Jaranowski, and B.S. Sathyaprakash (work in progress). 